\title{
Chronische Urtikaria: Viele behandeln sich lieber selbst
}

\author{
Eine Umfrage unter Patienten mit chronischer Urtikaria zeichnet ein düsteres Bild der \\ Versorgunglage in Deutschland. Viele, darunter drei von vier Langzeitpatienten, haben \\ es offenbar aufgegeben, auf ärztliche Hilfe zu hoffen.
}

Ein Team deutscher Dermatologen unter Führung von Marcus Maurer, Urtikariaspezialist der Berliner Charité, hat die Ergebnisse der Online-Umfrage ATTENTUS vorgelegt. Rund 17.500 Patienten mit chronischer Urtikaria (CU) hatten im Internet

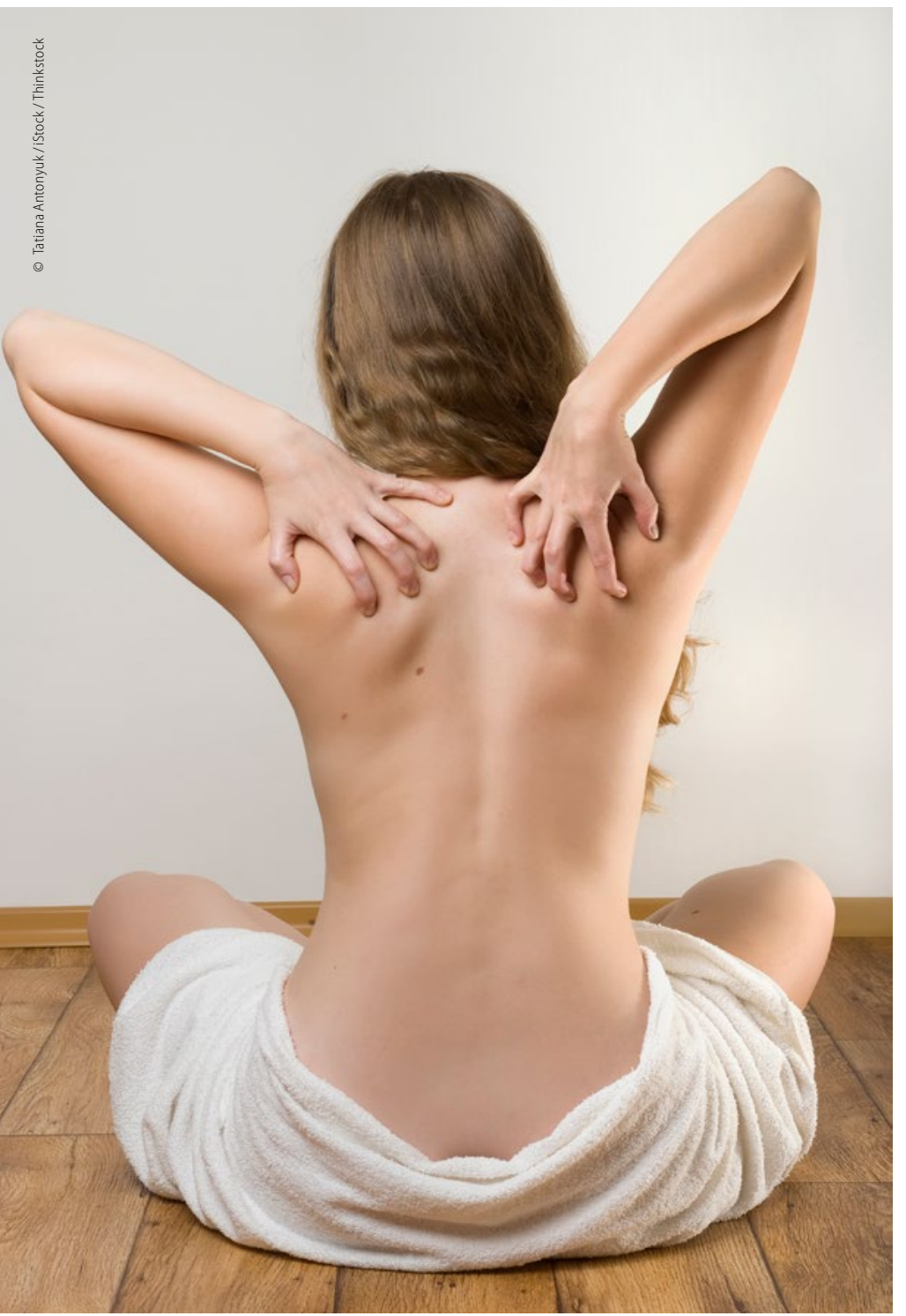

Vor allem langjährig erkrankte Patienten mit chronischer Urtikaria haben keinen Arztkontakt mehr und versuchen sich selbst zu behandeln.
Auskunft darüber gegeben, wie sie sich fühlen und welche medizinische Hilfe sie in Anspruch nehmen. Unter anderem konnten sie auf einer sechsstufigen Skala mit 6 als schlechtestem Wert angeben, wie sehr sie durch ihre Hauterkrankung im Alltag beeinträchtigt werden.

Ausgewertet haben Maurer und Kollegen nun die Angaben jener 9.055 Teilnehmer, die eine ärztliche CU-Diagnose erhalten hatten. Rund $80 \%$ waren Frauen. Im Durchschnitt waren die Befragungsteilnehmer im Alter von knapp 27 Jahren an CU erkrankt, die durchschnittliche Krankheitsdauer betrug 11,5 Jahre (bei einer Standardabweichung von 10,8 Jahren).

Etwa ein Drittel der Befragten gab an, aktuell durch die Urtikaria beeinträchtigt oder sehr beeinträchtigt zu sein (Werte von 5 oder 6 auf der Skala). Davon berichteten $38 \%$ über Symptome, die ganzjährig anhalten.

Nur $40 \%$ all derjenigen, die Beschwerden hatten, waren in ärztlicher Behandlung. Besonders trüb stellte sich die Situation der Patienten dar, die seit mindestens 15 Jahren an CU erkrankt waren. $74 \%$ von ihnen hatten keinen Arztkontakt mehr, sondern behandelten sich selbst - oder nahmen ihre Hautkrankheit einfach hin. Ebenso erging es einem Drittel jener, die das ganze Jahr über Symptome hatten. Mehr als die Hälfte äußerten, ein Arzt könne ihnen nicht helfen und/oder sie wüssten selbst am besten, wie sie ihre CU behandeln sollten.

Dabei war es nicht so, dass die Betroffenen nie einen Arzt gesehen hätten. $57 \%$ hatten einen Allgemeinarzt, $86 \%$ einen Dermatologen konsultiert. $42 \%$ waren sogar in einer Klinik vorstellig geworden. Dennoch gaben $17 \%$ an, Medikamente würden wenig oder gar nicht gegen ihre Beschwerden helfen, weitere $37 \%$ berichteten von unzureichend kontrollierten Symptomen. Mehr als jeder Zweite hatte bereits ein Kortikoid verschrieben bekommen, ohne dass dies den Alltag erleichtert hätte.

Eine Umfrage wie der vorliegende ATTENTUS-Survey lässt natürlich offen, ob es sich bei den Befragten um eine repräsentative Stichprobe von CU-Patienten handelt. Es ist nicht unwahrscheinlich, dass sich besonders die Meistgeplagten zu Wort gemeldet haben. Dennoch sehen Maurer und seine Mitarbeiter in den Ergebnissen Hinweise auf eine hochbelastete Gruppe von CU-Patienten, die keinen Zugang zu spezialisierten Zentren haben und diesen auch gar nicht mehr suchen. Zudem erhebe sich die Frage, „ob die Ärzte sich der CU-Therapieleitlinien völlig bewusst sind, die auf Symptomfreiheit für die meisten der Patienten ausgerichtet sind“.

Dr. Robert Bublak

Maurer M et al. Br J Dermatol 2016, online 4. Januar; doi: 10.1111/bjd.14203 